Letters to the Editor

\title{
Exchange Rate of Trimethyl Phosphate on Bis(trimethyl phosphate)- bis(thenoyltrifluoroacetonato)nickel(II) and -cobalt(II) as Measured by Phosphorus-31 Magnetic Resonance
}

\author{
Yoshiko Yano* and Akemi Takahashi** \\ *Laboratory of Chemistry, Wayo Women's University, Inchikawa 272 \\ **Institute of Chemistry, The University of Tsukuba, Sakura-mura, Ibaraki 305
}

\begin{abstract}
Kenwords Paramagnetic $\beta$-diketone chelate, phosphorus-31 NMR, trimethyl phosphate, solvation exchange rate, TTA adduct, ligand exchange
\end{abstract}

The solvent often coordinates to form an adduct with a metal complex which has an unsaturated coordination number. The adduct formation between some $\beta$-diketonato chelates and organic bases plays an important roles in synergistic solvent extraction. ${ }^{1-3}$ The typical bases are pyridines, tributyl phosphate (TBP), and alkylphosphine oxides. The authors reported about the solvent exchange on some bis- $\beta$-diketonatocobalt(II) and -nickel(II) complexes in pyridine. ${ }^{4}$ The result was that the stronger electron withdrawal of a substituent group on a chelate ring caused slower exchange because of the increase in the effective oxidation number of a central metal ion. It was qualitatively consistent with the results of other analogous complexes in pyridines ${ }^{5,6}$ and in dimethyl sulfoxide(DMSO) ${ }^{7}$ already reported. It is interesting that between pyridines and DMSO the solvent exchange rates were fairly different though the basicity of pyridines contributed to only small difference ${ }^{6,8}$ in the rates (Neither pyridine compared was necessary to be considered the steric effect.). This paper will describe the exchange of trimethyl phosphate (TMP) on $\mathrm{Ni}(\mathrm{tta})_{2}(\mathrm{tmp})_{2}$ and $\mathrm{Co}(\mathrm{tta})_{2}(\mathrm{tmp})_{2}$ with the one in the bulk solvent. TMP is not so practical as TBP in synergistic solvent extraction technique but the former gives less complicated NMR spectrum than the latter. Trimethyl phosphate has smaller donor number than both pyridine and DMSO.

\section{Experimental}

Materials
Trimethyl phosphate was fractionally distilled twice
under reduced pressure (b.p. $90.3^{\circ} \mathrm{C} / 19.8 \mathrm{mmHg}$. The
preparation of metal complexes was described previ-
${\text { ously. }{ }^{4} \text { Solutions for the NMR experiments were }}_{\text {prepared under a dry nitrogen atmosphere by dissolu- }}$
tion of the complexes into TMP which contained deuterated benzene. The concentrations of the paramagnetic ions were about $0.05 \mathrm{~mol} \mathrm{dm}^{-3}$.

\section{NMR measurements}

A JEOL FX-90Q FT-NMR spectrometer operated at $36.27 \mathrm{MHz}$ for ${ }^{31} \mathrm{P}$, was used in the ${ }^{2} \mathrm{D}$ lock mode. The rf power for the proton decoupling wás minimized so as to suppress the temperature raising in the probe.

All other experimental condition and apparatus used were essentially same as the previous work. ${ }^{4}$

\section{Results and Discussion}

The solvated complexes in TMP were characterized by means of electronic absorption spectra and the molecular weight measurement. The tta complexes in Table 1 insist themselves to be mononuclear species though the observed molecular weights include fairly large experimental errors because of the low vapour pressure of solvent even at $90^{\circ} \mathrm{C}$. The acetylacetonato (acac) complex of cobalt, on the other hand, has a value between a trimer and a tetramer in TMP while it had been identified to be a monomer in pyridine. ${ }^{4}$ The polymerized form is usual in solid state ${ }^{9,10}$ and in nonpolar solvents such as carbon tetrachloride. ${ }^{8}$ It seems that TMP solvates and forms a six-coordinate mononuclear complex only when the central metal ion has large effective oxidation number caused by the

Table 1 Molecular weight(M.W.) measurements in TMP by the method of vapor pressure depression

\begin{tabular}{lccc}
\hline Complex $^{\mathrm{a}}$ & Temp. $^{\circ} \mathrm{C}$ & M.W. obsd. & M.W. calcd. \\
\hline $\mathrm{Ni}(\mathrm{tta})_{2}$ & 94.6 & $505 \pm 33$ & 502 \\
$\mathrm{Co}(\mathrm{tta})_{2}$ & 89.0 & $436 \pm 31$ & 501 \\
$\mathrm{Co}(\mathrm{acac})_{2}$ & 89.0 & $814 \pm 58$ & 256 \\
\hline
\end{tabular}

a. No solvent molecule is taken into account. b. Assuming a respective monomer. 
strong electron withdrawal of the chelating tta ring. The difference in the behaviour between TMP and pyridine is probably explained by difference in Gutmann donicities $(\mathrm{DN})^{11}$, one of the indication of donor strength. For TMP, DN is 23.0 and for pyridine $33.1 \mathrm{kcal} / \mathrm{mol}$.

The temperature dependence of observed $T_{2 p}$ in Fig. 1 confirms to a typical solvent exchange in the whole range of temperature examined. The $T_{2 \mathrm{p}}$ is the transverse relaxation time of ${ }^{31} \mathrm{P}-\mathrm{NMR}$ of TMP due to the interaction with a paramagnetic complex and it is originally defined as $\left(T_{2}^{-1}-T_{2 A}{ }^{-1}\right)^{-1} P_{m}\left(1-P_{m}\right)^{-1} .12$ Here $T_{2}$ is the overall relaxation time of the paramagnetic solution and it is derived from the observed half-height width, $W\left(=\pi^{-1} T_{2}^{-1}\right)$ in $\mathrm{Hz}$, and $P_{\mathrm{m}}$ is the mole fraction of the solvent in the first coordination sphere. The $P_{\mathrm{m}}$ values were small enough to treat $\left(1-P_{m}\right)$ as unity. The $T_{2 \mathrm{~A}}{ }^{-1}$ is virtually zero since the observed line widths of pure TMP, $W_{\mathrm{A}}\left(=\pi^{-1} T_{2 \mathrm{~A}}{ }^{-1}\right)^{\prime} \mathrm{s}$, were negligibly small compared to $W$ 's. The present $T_{2 \mathrm{p}}$ is now re-defined as $(\pi W)^{-1} P_{\mathrm{m}}$. The solid lines in Fig. 1 are computed best fits, which produce the activation parameters and coupling constants, $A / h$ 's, listed in Table 2 . The computation $^{13}$ for the data reduction was essentially same as described previously. ${ }^{4}$ Figure 2 shows the temperature dependence of the chemical shift, $S_{\mathrm{p}}$, which is defined as $\left(S-S_{0}\right) P_{\mathrm{m}}{ }^{-1}$. Here $S$ and $S_{0}$ (both in $\mathrm{Hz}$ ) are the shift of the paramagnetic solution and the pure solvent respectively. The observed $S_{\mathrm{p}}$ 's in the

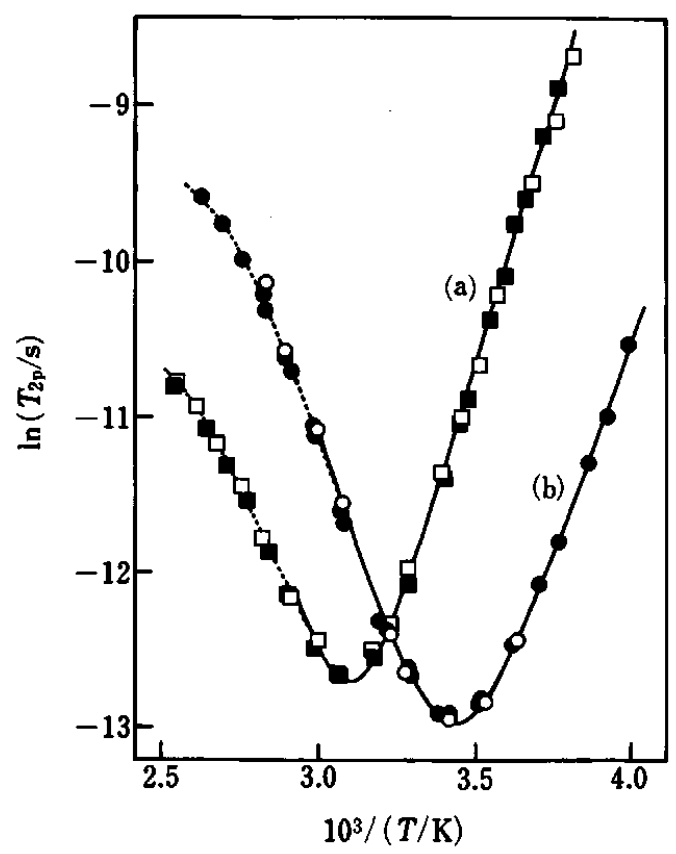

Fig. 1 Temperature dependence of the reduced P-31 NMR linewidth $\left(T_{2 p}\right)$ for the TMP solutions containing (a) $\mathrm{Ni}(\mathrm{tta})_{2}$ $(\mathrm{tmp})_{2}$ and (b) $\mathrm{Co}(\mathrm{tta})_{2}(\mathrm{tmp})_{2}$. The solutions contain 20( $\square$ and $O)$ and 50( $\square$ and $O)$ vol.\% of benzene. In the highest temperature range, the additional contribution from the fast exchange region ${ }^{1}$ is apparent.
Fig. 2 are interpreted self-consistently by the calculated solid lines from the parameters induced by the $T_{2 p}$ data. The calculation was mentioned in the previous paper. ${ }^{4}$ The self-consistence also appears in the $A / h$ values in Table 2. It should be also noted that dilution of trimethyl phosphate with benzene changed neither the temperature dependence of $T_{2 \mathrm{p}}$ nor that of $S_{\mathrm{p}}$ as can be seen in Figs. 1 and 2. It suggests any equilibrium is not necessary to be considered except the solvent exchange in question.

A solvent molecule bound to $\mathrm{Ni}(\mathrm{tta})_{2}$ exchanges about $\mathbf{1 7 0}$ times faster in TMP than in pyridine. ${ }^{4}$ The exchange rate in DMSO is estimated to be about 40 times faster than in pyridine. The estimation is based on the assumption that the solvent molecule bound to the tta complexes exchanges at a half rate of the case of the trifluoroacetylacetonato(tfa) complexes in DMSO as well as in pyridine.,14 The assumption seems reasonable since a linear correlation ${ }^{5,15}$ has been observed between $\log k_{1}(298 \mathrm{~K})$ and the sum of Taft parameters of substituents on chelating rings in different kinds of solvents. It is interesting to see the fact that the solvent exchange rate widely changes among pyridine, TMP, and DMSO. Further works would be needed to specify a factor which dominantly

Table 2 Kinetic parameters for TMP exchange

\begin{tabular}{|c|c|c|c|c|}
\hline Complex & $\begin{array}{c}\Delta H^{\ddagger} / \mathbf{k J a} \\
\mathrm{mol}^{-1}\end{array}$ & $\begin{array}{l}\Delta S^{\ddagger} / \mathrm{J}^{\mathrm{a}} \\
\mathrm{mol}^{-1} \mathrm{~K}^{-1}\end{array}$ & $\begin{array}{c}k_{1}(298 \mathrm{~K}) / \\
10^{-5} \mathrm{~s}^{-1}\end{array}$ & $\begin{array}{l}(A / h) / \\
\mathrm{MHz}\end{array}$ \\
\hline $\begin{array}{l}(\mathrm{tta})_{2}(\mathrm{tmp})_{2} \\
(\mathrm{tta})_{2}(\mathrm{tmp})_{2}\end{array}$ & $\begin{array}{l}52.5 \\
42.7 \\
\end{array}$ & $\begin{array}{l}28.1 \\
15.0\end{array}$ & 13 & $\begin{array}{l}8.1(8.7)^{b} \\
4.5(5.2)^{b}\end{array}$ \\
\hline \multicolumn{5}{|c|}{$\begin{array}{l}\text { a. } \Delta H^{\ddagger} / \mathrm{kJ} \mathrm{mol}^{-1}, \Delta S^{\ddagger} / \mathrm{J} \mathrm{mol}^{-1} \mathrm{~K}^{-1} \text { and } k_{1}(298 \mathrm{~K}) / \mathrm{s}^{-1} \\
\text { in pyridine exchange were } 48.6,-27.4 \text { and } 7.1 \times 10^{2} \text { for } \\
\mathrm{Ni}(\mathrm{tta})_{2}(\mathrm{py})_{2} \text { and } 48.3,2.6 \text { and } 2.9 \times 10^{4} \text { for } \mathrm{Co}(\mathrm{tta})_{2}(\mathrm{py})_{2}{ }^{4} \\
\text { b. Values in parentheses are from shift data (See ref. } 4) \text {. }\end{array}$} \\
\hline
\end{tabular}

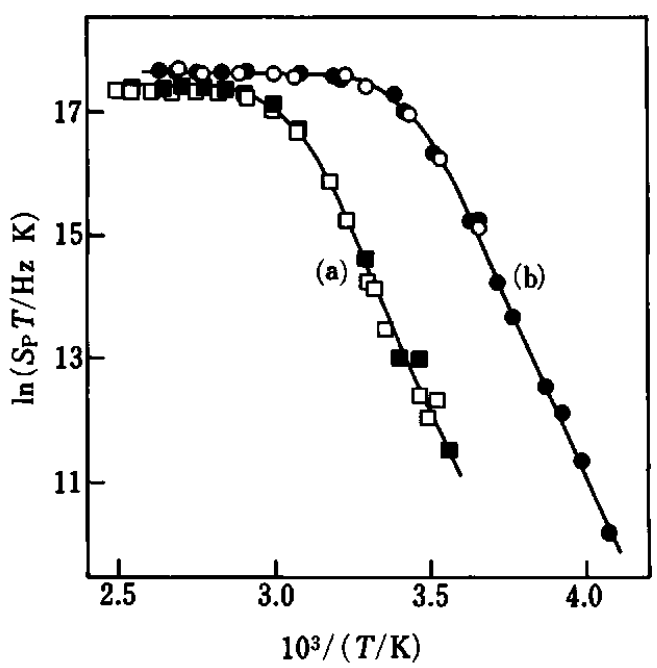

Fig. 2 Temperature dependence of the shift for the TMP solutions containing (a) $\mathrm{Ni}(\mathrm{tta})_{2}(\mathrm{tmp})_{2}$ and (b) $\mathrm{Co}(\mathrm{tta})_{2-}$ $(\mathrm{tmp})_{2}$. Symbols have the same meanings as in Fig. 1. 
controls the rate among the solvents. They are very different from each other in their features as the solvent. So far we merely suggest that the DN has a parallel relation to the rates. As the donicity of the solvent becomes smaller a bound solvent exchanges more rapidly with a free one (The DN for DMSO is 29.8. ${ }^{11}$ ). The donicity might be insufficient to discuss precisely about a minute change within analogous types of solvents. It is because the concept of the donicity does not take into account the steric effect, the contribution of the entropy term to the $\Delta G$ value of a solvation, and so on. In the case of $\operatorname{Co}(\operatorname{tta})_{2}(\mathrm{tmp})_{2}$ the observed $\Delta H^{\ddagger}$ value in Table 2 seems to reflect the smaller donicity of TMP than pyridine. For the nickel analogue, the $\Delta H^{\ddagger}$ value includes apparently any effect other than the donicity since the $\Delta S^{\ddagger}$ value is much bigger and the positive one compared to the pyridine exchange.

The authors would like to express their grateful acknowledgement to the late Prof. Kozo Nagashima of Institute of Chemistry, The University of Tsukuba, for his continuing support and encouragement during the course of this work. Thanks are also due to Mr. Hideto Suzuki for his assistance in NMR measurements.

\section{References}

1. H. Akaiwa, "Chushutsu-Bunri-Bunsekiho", Kodansha, Tokyo (1972).

2. T. Sekine and Y. Hasegawa, "Solvent Extraction Chemistry", Marcel Dekker, New York (1977).

3. Y. Yano, N. Kono, Y. Kondo and K. Nagashima, Nippon Kagaku Kaishi, 1974, 291.

4. S. Ito and Y. Yano, Bull. Chem. Soc. Jpn., 57, 2824 (1984).

5. J. Crea and S. F. Lincoln, Aust. J. Chem., 28, 1523 (1975).

6. R. W. Kluiber, R. Kukla, F. Thaller, J. Coord. Chem., 3, 39 (1973).

7. C. L. Watkins, M. E. Harris, J. Inorg. Nucl. Chem., 40, 1769 (1978).

8. M. J. Petrin and W. L. Reynolds, J. Inorg. Nucl. Chem., 33, 3978 (1971).

9. G. J. Bullen, R. Mason and P. Pauling, Inorg. Chem., 4, 456 (1965).

10. F. A. Cotton and R. H. Soderberg, Inog. Chem., 4, 1145 (1965).

11. V. Gutmann, "Coordination Chemistry in Non-Aqueous Solutions", p. 19, Springer, Vienna-New York (1968).

12. T. J. Swift and R. E. Connick, J. Chem. Phys., 37, 307 (1962); erratum, 41, 2553 (1964).

13. T. Nakagawa and Y. Oyanagi, "SALS", Univ. Tokyo Computer Centre (1979).

14. Y. Yano, A. Uejima, Unpublished data of $1: 4 \times 10^{3} \mathrm{~s}^{-1}$ for $k_{1}(298 \mathrm{~K})$ in pyridine exchange on $\mathrm{Ni}(\mathrm{tfa})_{2}(\mathrm{py})_{2}$.

15. R. W. Kluiber, R. Kukla, W. D. Horrocks, Inorg. Chem., 9, 1319 (1970). 